\title{
The Role of Interleukin-8 in the Infectious Process ${ }^{a}$
}

\author{
STEVEN L. KUNKEL, ${ }^{b, c}$ NICKOLAS W. LUKACS, ${ }^{b}$ AND \\ ROBERT M. STRIETER ${ }^{d, e}$ \\ Departments of Pathology ${ }^{b}$ and Internal Medicine ${ }^{d}$ \\ Division of Pulmonary and Critical Care Medicine $e^{e}$ \\ The University of Michigan Medical School \\ Ann Arbor, Michigan 48109-0602
}

The recruitment of blood-born leukocytes to a specific site of infection is one of the most fundamental of all inflammatory processes. To successfully elicit the appropriate leukocyte populations, a number of dynamic alterations must first occur in a tightly coordinated manner. The first changes that are evident involve alterations in the endothelium as this structure is converted from a passive to an active role in inflammation. ${ }^{1,2}$ The changes that occur in the endothelium are important in localizing the circulating leukocytes to a restricted area of injury where the infectious process is originating. The actual mechanism(s) that leads to the generation of an "inflamed" or activated endothelium is likely multifactoral. Bacteria-derived products, such as lipopolysaccharide (LPS), can serve as powerful agents for stimulating endothelial cells. ${ }^{2}$ In addition, early response cytokines, including interleukin-1 alpha (IL-1 $\alpha$ ), interleukin-1 beta (IL-1 $\beta$ ), and tumor necrosis factor alpha (TNF $\alpha$ ), are also classic mediators that can activate the endothelium.' Interestingly, LPS can either directly stimulate or indirectly activate endothelial cells by induction of resident, tissue macrophage-derived IL-1 or TNF. ${ }^{3,4}$ Expression of these cell-derived mediators can establish a cytokine network necessary to fully drive the inflammatory response. ${ }^{5,6}$ Independent of an exogenous (LPS) or endogenous (IL-1 or TNF) signal, activation of the endothelium results in the expression of adhesion molecules on the surface of endothelial cells and leukocytes. The net result of this interaction is the localization of specific leukocyte populations to an area of tissue injury. Elegant studies have demonstrated that the endothelial cell-leukocyte adhesion process is comprised of discrete steps, which include an initial "rolling" event followed by tenacious binding by strong leukocyte-to-endothelial cell interactions. Once tethered to endothelial cells in a local area of tissue injury, the leukocyte must next traffic out of the vessel lumen, move through the basement membrane, and successfully arrive at the inflammatory site. Thus, binding of leukocytes to the endothelium is a transient event.

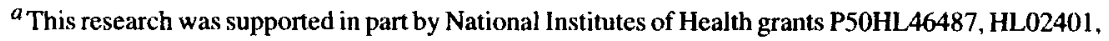
HL31693, and HL35276 and a grant from the Tobacco Research Council.

${ }^{c}$ Address for correspondence: Steven L. Kunkel, Ph.D., Department of Pathology, 1301 Catherine Road/Box 0602, The University of Michigan Medical School, Ann Arbor, Michigan 48109-0602.
} 


\section{CHEMOTACTIC FACTORS}

Whereas events involved in the initial binding of leukocytes to an activated endothelium are becoming increasingly clear, the actual movement of certain leukocyte populations from the vasculature to an exact site of tissue injury is not well understood. However, recent information suggests that specific targeting and movement of leukocytes involve the further participation of adhesion molecules and chemotactic factors often expressed by stromal and other resident tissue cells. The idea that leukocytes follow chemical signals is not new, as historic studies have identified chemotactic factors that can induce the directed migration of leukocytes. For example, bacterial peptides (fmetleuphe), polypeptides (C5a), and lipids (LTB and PAF) have all demonstrated the ability to elicit various leukocyte populations. ${ }^{7-9}$ There is little doubt that these chemotactic factors play an important role in the initiation of leukocyte recruitment; however, their transient expression, instability, and lack of cell specificity have always left a void in the total explanation of the chemotactic response. The finding that many chemotactic factors possess little specificity for eliciting specific leukocyte populations has been inconsistent with the histopathology of a number of diseases. For example, acute inflammatory reactions are often characterized by the presence of polymorphonuclear leukocytes, whereas other chronic inflammatory diseases are characterized by mononuclear cell infiltration. Further understanding of the mechanism of this biologic response has been attained by studies assessing the role of chemotactic cytokines or chemokines in mediating leukocyte elicitation. ${ }^{(0-12}$

The first chemotactic cytokine to be discovered was originally termed monocytederived neutrophil chemotactic factor (MDNCF) and later classified as interleukin8 (IL-8). ${ }^{13}$ Subsequent studies proved that most nucleated cells, when appropriately stimulated, have the capacity to generate $1 \mathrm{~L}-8 .^{14-17}$ As research in the chemotactic cytokine arena progressed, it became increasingly apparent that IL-8 was only one member of a supergene family that possesses chemotactic activity for polymorphonuclear (PMN) leukocytes. ${ }^{10}$ Presently, chemokines that possess chemotactic activity for PMNs include epithelial cell-derived neutrophil activating peptide (ENA-78), neutrophil-activating peptide-2 (NAP-2), growth-related oncogene (GRO-alpha, beta, and gamma), and macrophage inflammatory protein-2 alpha and beta (MIP-2 $\alpha$ and $\beta$ ). This supergene family of chemotactic cytokines is structurally characterized by the conserved location of two of four cysteine residues. The first two cysteines are separated by one additional amino acid; therefore, this family is often referred to as the C-X-C chemokine family. The aforementioned chemotactic factors also share a common amino acid motif, ELR (glutamic acid, leucine, and arginine), which is present in juxtaposition to the first $\mathrm{NH}_{2}$-terminal cysteine amino of the $\mathrm{C}-\mathrm{X}$-C. ${ }^{10}$ The importance of the ELR is that this sequence is responsible for neutrophil activation via receptor ligand interactions. Interestingly, other $\mathrm{C}-\mathrm{X}$-C supergene family members that do not possess the ELR motif are not chemotactic for neutrophils. These polypeptides include platelet factor-4 (PF-4) and an interferon-inducible protein (IP-10). Recent investigations demonstrated that generation of recombinant PF-4, which has been mutated to include the ELR, is indeed chemotactic for neutrophils. On the contrary, ELR-containing chemokines that have been truncated to remove the ELR element dramatically change their binding affinity for the appropriate receptor, subsequently reducing their chemotactic activity. However, the synthesis of short peptides 
that include the ELR motif is not active, suggesting that interaction of other domains of the chemokine molecule is necessary for biologic activity. The receptor system for the C-X-C chemokines is comprised of two high affinity receptors. One is promiscuous and able to interact with other family members, whereas the other receptor possesses specificity for an individual chemokine. The chemokine receptors are seven transmembrane-spanning proteins that signal via GTP-binding proteins.

Whereas the IL-8 or C-X-C supergene family possesses chemotactic activity mainly for neutrophils, a related polypepide family has been discovered with activity predominantly for mononuclear cells. This class of chemokines is also structurally identified by the location of two of four cysteines. However, the first two cysteines are in juxtaposition and this family is designated as the C-C chemokine family." Monocyte chemoattractant protein-1, 2, and 3 (MCP-1, MCP-2, and MCP-3), macrophage inflammatory protein-1 (MIP-1) alpha and beta, and RANTES are members of this family of chemotactic cytokines. Recent data suggest that the ligand:receptor system for the C-C family is similar to the C-X-C system. Structure/function analysis of these chemokines and the quest for receptor antagonists have greatly accelerated interest in this area of inflammation.

\section{EXPRESSION AND REGULATION OF CHEMOTACTIC CYTOKINES}

A striking novel aspect of both the C-C and C-X-C chemokine families is the expression pattern that results in the generation of these mediators. Both inflammatory and noninflammatory cells have been identified as cell sources of these chemotactic factors. Thus, neutrophils, monocytes, smooth muscle cells, fibroblasts, epithelial cells, and endothelial cells have all been identified as rich sources of chemokines, especially IL-8. ${ }^{14-17}$ Although these cells do not usually generate high levels of IL-8 during resting conditions, they can produce elevated concentrations when stimulated with an appropriate agonist. One exception is the expression of IL-8 by certain tumor cell lines which possess the ability to constitutively generate significant levels of IL-8. Two of the most potent agents for the induction of IL-8 are IL-1 and TNF. These early response cytokines induce the production of IL- 8 by most nucleated cells at picomolar to nanomolar concentrations. Although IL-1 and TNF are potent early response cytokines that are able to induce IL-8 expression, other cytokines such as interleukin-6 (IL-6) do not serve as effective signals for the production of IL-8. Thus, expression of IL-8 depends on both cell and stimulus specificity. ${ }^{56}$ LPS and other bacteria-derived products can also serve as important activating agents for the production of IL-8; however, LPS-dependent IL-8 production is not routinely observed from all cells. Although both mononuclear phagocytes and polymorphonuclear leukocytes can generate IL-8 in response to LPS, most noninflammatory cells do not generate IL-8 in response to bacteria-derived products.

The inability of LPS to activate specific cells to produce IL-8 does not imply that Gram-negative bacteria are not involved in the process of generating chemotactic cytokines. Evolving scientific information suggests that generation of cytokine networks via LPS-dependent mechanisms is a major pathway for the production of chemokines. ${ }^{5.6}$ During the pathogenesis of an infectious process, it is likely that 
bacterial products activate sentinel, resident macrophages which are ubiquitously dispersed throughout tissue. This activation event results in the production of early response cytokines (IL-1 and TNF), which can "network" with surrounding resident tissue cells and orchestrate the production of more distal cytokines, including IL-8. Therefore, the overall effect of the inciting agent is to activate the inflammatory cascade and cause the up-regulation of appropriate chemotactic factors to insure the successful elicitation of leukocytes to the area of inflammation. Interestingly, noninflammatory cells such as stromal and epithelial cells can no longer be only designated as targets of the inflammatory response, but are more likely to be considered as effector cells via the expression of important cytokines. This scenario was previously shown to be active in the generation of lung and hepatic chemotactic cytokines using in vitro models. For example, the conditioned media from LPS-challenged alveolar macrophages, recovered from nonsmoking, disease-free volunteers, possessed significant activity in stimulating the expression of IL-8 from pulmonary fibroblasts. ${ }^{5}$ These same lung fibroblasts were not susceptible to LPS stimulation, suggesting that factors(s) in the alveolar macrophage-conditioned media were providing the signal for noninflammatory cell activation. Similar results were observed using type II-like pneumocyte cell lines challenged with conditioned media from LPS-treated alveolar macrophages. ${ }^{6}$ Further studies identified that both IL-1 and TNF are involved in the expression of IL-8 from these noninflammatory cells. In these studies, conditioned media from alveolar macrophages treated with neutralizing antibodies to IL-1 or TNF significantly reduced the production of fibroblast-derived IL-8. This networking phenomenon is not restricted only to the lungs, as previous studies demonstrated that cytokine networking can also occur in the liver. These investigations showed that LPS-activated Kupffer cells can secrete both IL-1 and TNF which can interact with hepatocytes and induce the production of IL-8 from these noninflammatory cells. It is becoming increasingly clear that this pathway of cell activation leading to the production of a potent chemotactic factor for neutrophils is likely a mechanism for leukocyte elicitation common to all tissues. Stimulated resident macrophages signal the surrounding normal resident cells to produce a chemoattractant that elicits a specific population of leukocytes to a local area of inflammation. This scenario is a key component of leukocyte elicitation, as it insures that the recruitment response is timely, specific, and localized only to the area of tissue injury. This latter phenomenon is one of the most fundamental aspects of normal inflammation.

\section{NEUTROPHIL-DEPENDENT CHEMOKINE EXPRESSION}

In acute inflammation, the first blood-born leukocyte to arrive at a site of tissue injury or infection is the neutrophil. Historically, these elicited cells have been important in clearing infectious agents by the release of proteolytic enzymes and reactive oxygen metabolites. In addition, these terminally differentiated leukocytes have classically been viewed as possessing limited protein-synthesizing activity. Recent studies showed that neutrophils can produce polypeptides, including heat shock proteins, elastase, plasminogen activator, cytoskeletal proteins, and cell surface receptors. ${ }^{18-20}$ Also, stimulated neutrophils have the ability to generate cytokines, that 
are important in maintaining the inflammatory response, such as colony-stimulating factors (G-CSF and GM-CSF), interferon-alpha, interleukin-6, interleukin-1, tumor necrosis factor, and interleukin- $8 .{ }^{21-26}$ The production of these polypeptide mediators at a site of injury is likely to play a significant role in the subsequent evolution of inflammation by both paracrine and autocrine interactions, involving other leukocytes and/or noninflammatory cells.

Investigations have demonstrated that neutrophils are capable of generating IL-8 in response to both endogenous and exogenous signals. For example, the phagocytic response to zymosan or other particulate material, as well as LPS, may serve as an exogenous agent for neutrophil-derived IL-8. In addition, the adherence response is a significant signal which may lead to the production of IL-8. The mechanism(s) involved in adherence-dependent IL-8 expression may be an adhesion moleculemediated event, as freshly isolated neutrophils in suspension did not express steadystate levels of IL-8 mRNA or release antigenic IL-8. The concomitant treatment of neutrophils with actinomycin D also blocked any adherence-induced IL-8 mRNA and protein. Subsequent investigations showed that adherence-induced, neutrophilderived IL-8 is under the regulation of repressor proteins. This was ascertained by the concomitant addition of cycloheximide to neutrophils undergoing adherence, resulting in the superinduction of IL-8 mRNA.

Whereas exogenous stimuli can cause neutrophils to generate IL-8, other endogenous mediators are highly effective in generating neutrophil-derived IL-8. In particular, IL-1 and TNF were effective stimulating agents for neutrophils in both a timeand dose-dependent manner. The findings that $1 \mathrm{~L}-1$ can stimulate neutrophil-derived IL-8 is intriguing in light of reports demonstrating that IL-1 is not a potent neutrophilactivating compound for direct chemotaxis, exocytosis, or enzyme release. However, IL-1 can induce neutrophils to generate a potent neutrophil chemoattractant, which is likely to perpetuate the recruitment process. Other factors have also been identified as important neutrophil-activating agents and play both direct and indirect roles in leukocyte recruitment. C5a, $\mathrm{AMLP}$, and $\mathrm{LTB}_{4}$ are all known mediators of neutrophil recruitment that also synergize in the presence of LPS to induce neutrophil-derived IL-8. The synergistic activity of bacteria-derived products plus traditional neutrophilactivating agents such as C5a, fMLP, and $\mathrm{LTB}_{4}$ could aid in explaining the rapid elicitation of neutrophils to a site of bacterial infection. At the inflammatory lesion, neutrophils can encounter host-derived cytokines (IL-1 and TNF), endogenous chemotactic and activating agents (C5a, fMLP, and $\left.\mathrm{LTB}_{4}\right)$, as well as bacteria and their products, which can stimulate neutrophils in a synergistic manner for the production of neutrophil-derived IL-8. Although the aforementioned classic neutrophil chemotactic factors (C5a, fMLP, and $\mathrm{LTB}_{4}$ ) are indeed chemotactic agents, these agonsts have relatively short half-lives. Thus, local generation of IL-8 by mononuclear phagocytes, noninflammatory cells, and neutrophils may sustain the inflammatory response through continued neutrophil recruitment and activation. The importance of the neutrophil as an effector cell via de novo cytokine production places this inflammatory cell in a pivotal position to aid in the orchestration of conventional immune responses.

Although increasing evidence supports the role of the neutrophil as an important cytokine-producing leukocyte, less is known about the mechanism(s) that regulates the expression of these neutrophil-derived polypeptides. Interestingly, specific com- 
pounds that control the production of monocyte-derived cytokines also appear to have a regulating influence on neutrophil-derived cytokines.

Dexamethasone, interleukin-4 (IL-4), and interleukin-10 (IL-10) have been demonstrated in a time- and dose-dependent manner and in a delayed addition manner to regulate the production of neutrophil-derived IL-8. Neutrophils treated with LPS and dexamethasone at 100, 10, and $1 \mathrm{nM}$ demonstrated a $73 \%, 47 \%$, and $45 \%$ reduction, respectively, in IL-8 levels. Concentrations of dexamethasone less than 1 $\mathrm{nM}$ failed to cause any further suppression of neutrophil IL-8 in response to LPS. In similar studies, IL-4 and IL-10 exerted a suppressive effect on neutrophil IL-8 production over a concentration range of $100 \mathrm{pg} / \mathrm{ml}$ to $100 \mathrm{ng} / \mathrm{ml}$. Further studies demonstrated that the delayed addition of dexamethasone, IL-4, or IL-10 could still dramatically influence the production of neutrophil-derived IL-8. The production of IL-8 from neutrophils stimulated with LPS at time 0 and given either of the three suppressing agents 2 hours later was still significantly inhibited at the final 18 -hour time-point. Additional studies demonstrated that the suppressive effects of IL-4, IL-10, and dexamethasone were at the transcriptional level. Northern blot analyses of neutrophils challenged with LPS plus one of the three immunomodulating agents showed that steady-state levels of IL-8 were significantly reduced as compared to LPS alone. These studies are significant in that they demonstrate the regulation of neutrophil-derived IL-8 at the transcriptional level.

The foregoing investigations are of interest, because IL- 4 and IL-10 are phenotypically expressed by $\mathrm{TH} 2$ lymphocytes and have been shown to possess pleiotropic activities in a number of cell systems. Although IL-4 was originally described as a B- and T-cell maturation and growth factor, it has also been reported to have the dual role of either enhancing or suppressing monocyte functions. Interleukin-4 can also enhance monocyte major histocompatibility complex expression and augment tumoricidal activity. In contrast, IL-4 has been shown to block the generation of monocyte-derived cytokines, including IL-6, IL-8, TNF, and IL-1, as well as to reduce the generation of reactive oxygen metabolites. Whereas IL-4 can inhibit important phagocyte functions, it appears to be a general activating agent for endothelial cells. This cytokine can stimulate endothelial cells to induce the expression of specific adhesion molecules for lymphocytes and monocytes but not neutrophils, suggesting that $\mathrm{IL}-4$ is important in driving the switch from an acute to a chronic inflammatory response. These studies demonstrating a reduction of IL-8 by IL-4-treated neutrophils support this hypothesis, as the continued generation of IL-8 would propagate the acute inflammatory response by continued neutrophil recruitment.

Interleukin-10 may mechanistically play a key role in the leukocyte switch in a manner similar to that of IL-4. Originally, IL-10 was found to be able to inhibit the production of gamma interferon from $\mathrm{CD}^{+} \mathrm{T}$ cells; however, this cytokine can also suppress a number of cytokines necessary to maintain an inflammatory response. Monocytes treated with LPS in the presence of graded doses of IL-10 produced significantly less IL-1, TNF, IL-6, IL-8, and G-CSF. In addition, IL-10 can suppress macrophage cytotoxic activity, parasite killing, and macrophage-derived nitric oxide production. Thus, the cytokine networks that function in both a positive and negative manner for the production and regulation of IL-8 are an important yet complex system. 


\section{BACTERIAL INFECTIONS AND IL-8 EXPRESSION}

Cytokines unquestionably represent an important communication link necessary to combat infectious processes. Previous studies identified cytokines, such as TNF, IL-1, and IL-6, as being elevated in infectious disease states and suggested that they are indicators of clinical severity. Although these cytokines undoubtedly play a role in disease progression, other chemotactic cytokines appear to be involved in the initiation and maintenance of bacterial infection. Elevated concentrations of IL-8 were identified after injection of intravenous Escherichia coli in primates and after an injection of LPS into human volunteers. ${ }^{27,28}$ The kinetics of the in vivo expression pattern for IL-8 was similar to that for IL-6 in that the detection of IL-8 was delayed about 3 hours postchallenge. These data suggest that in vivo cytokine networking could account for the mechanism of IL-8 production, because IL- 8 production was distal to the acute production of TNF in these experiments.

Recently, IL-8 levels were identified in human patients with either severe bacterial infections, septicemia, or adult respiratory distress syndrome. A detailed longitudinal study assessing the plasma concentrations of TNF, IL-6, and IL-8 was reported for patients with melioidosis. ${ }^{29}$ This infection is caused by Pseudomonas pseudomallei and is endemic throughout Southeast Asia. Clinically, melioidosis caused a serious septicemic illness with a $40 \%$ death rate. In this study, 18 patients were monitored for circulating levels of plasma IL-8, IL-6, and TNF. Elevated concentrations of plasma IL-8 were found in 8 of 18 subjects, including four who subsequently died. Three of the four who died had the highest levels of IL-8, 161-362 pg/ml; therefore, IL-8 levels may have value as a predictor of mortality. Qualitative plasma IL-6 levels followed a pattern similar to those of IL-8 in that the highest levels of IL-6 were found in three of the four patients who died of this disease. On the contrary, no correlation was noted between plasma TNF levels and concentrations of IL-8 or IL-6. Additional clinical studies demonstrated a close correlation between plasma levels of TNF, IL-6, and IL-8 and the pathophysiology of the Jarisch-Herxheimer reaction (J-HR) associated with the treatment protocol for certain infectious diseases. ${ }^{30}$ In this clinical study, 14 of 17 patients (82\%) diagnosed with relapsing fever due to Borrelia recurrentis and treated with penicillin subsequently experienced J-HR. This acute reaction is characterized by rigors, fever, leukopenia, and a decrease in mean arterial blood pressure. Although no fatalities occurred, plasma TNF, IL-6, and IL-8 levels rose seven, six, and fourfold, respectively, over admission levels. Interestingly, a pulsatile release of the three different cytokines was associated with J-HR which occurred between 2 and 4 hours after antibiotic treatment. The peak values for TNF, IL-6, and IL-8 were $126 \pm 38,9,578 \pm 1,808$, and 8,102 $\pm 4,491 \mathrm{pg} / \mathrm{ml}$, respectively.

In further studies, 105 of 151 patients undergoing mechanical ventilation in an intensive care unit had detectable IL-8 levels in bronchial secretions. ${ }^{31}$ However, IL-8 levels could not be detected in arterial blood, mixed venous blood, or urine samples. The occurrence of IL-8 in bronchial secretions of the pulmonary air space was significantly associated with patients with multiple injuries and nosocomial pneumonia. Interestingly, $66 \%$ of the patients who had positive IL-8 levels were diagnosed as having nosocomial pneumonia. Most of the patients tested positive for IL-8 within the first 36 hours of admission, suggesting that the production of IL-8 
was a relatively rapid event. In addition, detectable IL-8 levels were associated with significant pulmonary dysfunction, as evidenced by static lung compliance and a low $\mathrm{PaO}_{2} / \mathrm{FIO}_{2}$ ratio.

Finally, recent investigation of 29 patients with adult respiratory distress syndrome (ARDS) demonstrated a significant correlation between the early appearance of IL-8 in the bronchoalveolar lavage (BAL) fluid and the development of clinical ARDS ${ }^{32}$ The 29 patients were grouped into three at-risk categories: multiple trauma, perforated bowel, and pancreatitis. Both BAL and plasma from each patient were assessed for antigenic IL-8 within a mean of 2 hours of trauma, 34 hours of diagnosed pancreatitis, and 33 hours of diagnosed perforated bowel. Although no significant difference was noted between the groups in plasma IL-8 levels, a significant difference was found in the BAL fluid IL-8 levels in the group that progressed to ARDS as compared to the non-ARDS group $(p$ value $=0.0006$ ). Patients with clinical ARDS had IL-8 levels of $3.06 \mathrm{ng} / \mathrm{ml}$ versus $0.53 \mathrm{ng} / \mathrm{ml}$ in the non-ARDS group.

Immunocytochemical analyses for antigenic IL-8 demonstrated more intense staining of alveolar macrophages from patients who subsequently progressed to ARDS than of alveolar macrophages from non-ARDS patients. The latter findings support the concept that activated alveolar macrophages are a potent source of cytokines, such as IL-8, during the initiation of ARDS. These findings suggest that measurement of IL-8 levels in the BAL fluid of at-risk patients could aid in early identification of those likely to progress to ARDS.

\section{REFERENCES}

1. Pober, J. S., P. Bevilacqua, D. L. Mendrick, L. A. Lapierre, W. Fiers \& M. A. Gimbrone. 1986. Two distinct monokines, interleukin-1 and tumor necrosis factor, each independently induce biosynthesis and transient expression of the same antigen on the surface of cultured human vascular endothelial cells. J. Immunol. 137: 16801693.

2. Cybulsky, M. I., M. K. W. Chan \& H. Z. Movat. 1988. Acute inflammation and microthrombosis induced by endotoxin, interleukin-1, and tumor necrosis factor, and their implication in gram negative infection. Lab. Invest. 58: 365-378.

3. Chensue, W., P. D. Terebuh, D. G. Remick, W. E. Scales \& S. L. Kunkel. 1991. In vivo biologic and immunohistochemical analysis of interleukin- 1 alpha, beta, and tumor necrosis factor during experimental enotoxemia: Kinetics, Kupffer cell expression and glucocorticoid effects. Am. J. Pathol. 138: 395-402.

4. Strieter, R. M., D. G. Remick, J. P. LyNCh \& S. L. KUnKel. 1989. Differential regulation of tumor necrosis factor-alpha in human alveolar macrophages and peripheral blood monocytes: A cellular and molecular analysis. Am. J. Respir. Cell Mol. Biol. 1: 57 63.

5. Rolfe, M. W., S. L. Kunkel, T. J. Standiford \& R. M. Strieter. 1992. Pulmonary fibroblasts expression of interleukin-8: A model for alveolar macrophage derived cytokine networking. Am. J. Respir. Cell Mol. Biol. 5: 579-585.

6. Standiford, T. J., S. L. KunKel, M. A. Basha \& R. M. Strieter. 1990. Interleukin-8 expression by pulmonary epithelial cells: A model for cytokine networks in the lung. J. Clin. Invest. 86: 1945-1953.

7. Fernandez, H. N., P. M. Henson, A. Otani \& T. E. Hugli. 1978. Chemotactic response to human C3a and C5a anaphylatoxins. 1. Evaluation of C3a and C5a leukotaxis in vitro and under stimulated in vivo conditions. J. Immunol. 120: 109-115. 
8. Ford-Hutchinson, A. W., M. A. Bray, M. V. Doig \& M. J. Smith. 1980. Leukotriene B4 a potent chemokinetic and aggregating substance released from polymorphonuclear leukocytes. Nature 286: 262-265.

9. Shiffman, E. B. A. Corcoran, S. M. Wahl, H. J. Showell \& E. Becker. 1975. Nformylmethionyl peptides as chemoattractants for leukocytes. Proc. Natl. Acad. Sci. USA 72: 1059 - 1062 .

10. Matsushima K. \& J. J. Oppenheim. 1990. Interleukin-8 and MCAF: Novel inflammatory cytokines inducible by IL-1 and TNF. Cytokine 1: 2-13.

11. Leonard, E. J. \& T. Yoshimura. 1990. Human monocyte chemoattractant protein-1 (MCP-1). Immunol. Today 11: 97-100.

12. Strieter, R. M., S. L. Kunkel, H. J. Showell, D. G. Remick, S. H. Phan, P. A. Ward \& R. M. MARKs. 1989. Endothelial cell gene expression of a neutrophil chemotactic factor by TNF-alpha, LPS, and IL-1 beta. Science 243: 1467-1469.

13. Matsushima, K., K. Moishita, T. Yoshimura, S. Lavu, Y. Obayashi, W. Lew, E. APPELla, E. J. LeONARD \& J. J. OPPENHEIM. 1988. Molecular cloning of human monocyte-derived neutrophil chemotactic factor (MDNCF) and induction of MDNCF by interleukin-1 and tumor necrosis factor. J. Exp. Med. 167: 1883-1893.

14. Thornton, A. J., J. Ham \& S. L. Kunkel. 1991. Kupffer cell-derived cytokines induce the synthesis of a leukocyte chemotactic peptide, interleukin-8, in human hepatoma and primary heptocyte cultures. Hepatology 14: 1112-1122.

15. Schmouder, R. L., R. M. Strieter, R. C. Wiggins \& S. L. Kunkel. 1992. In vitro and in vivo interleukin-8 production in human renal cortical epithelia. Kidney Int. 41: 191198.

16. Strieter, R. M., S. H. Phan, H. J. Showell, D. G. Remick, J. P. Lynch, R. M. Marks \& S. L. KUNKEL. 1989. Monokine-induced neutrophil chemotactic factor gene expression in human fibroblasts. J. Biol. Chem. 264: $10621-10626$.

17. DibB, C. R., R. M. STRIETER, M. Burdick \& S. L. Kunkel. 1992. Expression of interleukin8 by LPS-stimulated bone marrow derived mononuclear cells. Infect. Immunol. 60: 3052 - 3058.

18. Granelli-Piperno, A., J. D. Vassalli \& E. Reich. 1979. RNA and protein synthesis in human polymorphonuclear leukocytes. J. Exp. Med. 149: 284-289.

19. JACK, R. M. \& D. T. FEARON. 1988. Selective synthesis of mRNA and proteins by human peripheral blood neutrophils. J. Immunol. 140: 4286-4293.

20. Eid, N. S., R. E. KRAVATH \& K. W. LANKS. 1987. Heat-shock protein synthesis by human polymorphonuclear cells. J. Exp. Med. 165: 1148-1153.

21. Dubravec, D. B., D. R. Spriggs, J. A. Mannick \& M. L. Rodrick. 1990. Circulating human peripheral blood granulocytes synthesize and secrete tumor necrosis factoralpha. Proc. Natl. Acad. Sci. USA 87: 6758-6761.

22. Tiku, K., M. L. Tiku \& J. L. Skosey. 1986. Interleukin-l production by human polymorphonuclear neutrophils. J. Immunol. 136: 3677-3685.

23. Lindemann A., D. Riedel, W. Oster, S. C. Meuer, D. Blohm, R. H. Mertelsmann \& F. HerrmanN. 1988. Granulocyte/macrophage colony-stimulating factor induces interleukin-1 production by human polymorphonuclear neutrophils. J. Immunol. 140: $837-839$.

24. Cicco, N. A., A. Lindemann, J. Content, P. Vandenbussche, M. Lubbert, J. Gauss, R. H. Mertelsmann \& F. Herrmann. 1990. Inducible production of interleukin-6 by human polymorphonuclear neutrophils. Blood 75: 2049-2052.

25. Strieter, R. M., K. Kasahara, R. Allen, H. J. Showell, T. J. Standford \& S. L. KUNKEL. 1990. Human neutrophils exhibit disparate chemotactic factor gene expression. Biochem. Biophys. Res. Comm. 173: 725-730. 
26. Strieter, R. M., K. Kasahara, R. A. Allen, T. J. Standiford, M. W. Rolfe, F. S. Becker, S. W. Chensue \& S. L. Kunkel. 1992. Cytokine-induced neutrophil-derived interleukin-8. Am. J. Pathol. 141: 397-407.

27. Martich, G., R. L. Danner, M. Ceska \& A. F. Suffredini. 1991. Detection of interleukin8 and tumor necrosis factor in normal humans after intravenous endotoxin: The effect of antiinflammatory agents. J. Exp. Med. 173: $1021-1024$.

28. van Zee, K. J., L. E. DeForge, E. Fischer, M. A. Marano, J. S. Kenney, D. G. Remick, S. F. Lowry \& L. L. Moldawer. 1991. IL-8 in septic shock, endotoxemia, and after IL-1 administration. J. Immunol. 146: 3478-3482.

29. Friedland, J. S., Y. Suputtamongkol, D. G. Remick, W. Chaowagul, R. M. Strieter, S. L. Kunkel, N. J. White \& G. E. Griffin. 1992. Prolonged elevation of interleukin8 and interleukin- 6 concentrations in plasma and of leukocyte interleukin-8 mRNA levels during septicemic and localized Pseudomonas pseudomallei infection. Infect. Immunol. 60: 2402- 2408.

30. Negussie, Y., D. G. Remick, L. E. DeForge, A. Eynon, S. L. Kunkel \& G. E. Griffin. 1992. Detection of plasma tumor necrosis factor, interleukin-6, and interleukin-8 during the Jarisch-Herxheimer reaction of relapsing fever. J. Exp. Med. 127: 1207-1212.

31. Rodriguez, J. L., C. G. Miller, L. E. DeForge, L. Kelty, S. J. Shanley, R. H. Barlett \& D. G. Remick. 1992. Local production of interleukin-8 is associated with nosocomial pneumonia. J. Trauma 33: 74-82.

32. Donnelly, S. C., R. M. Strieter, S. L. Kunkel, A. Walz, C. R. Robertson, D. C. Carter, I. S. Grant, A. J. Pollok \& C. Haslett. 1993. Interleukin-8 and development of adult respiratory distress syndrome in at-risk patient groups. Lancet 341: 643-647. 